\title{
Alternatives to the frequency control of an islanded wind farm
}

\author{
I. Zubia ${ }^{1}, X$. Ostolaza $^{2}$ and L.M. Bandrés ${ }^{1}$ \\ ${ }^{1}$ Department of Electrical Engineering \\ University of the Basque Country \\ Plaza Europa 1, 20018 San Sebastián (Spain) \\ phone:+34 943 017238, fax:+34 943 017130, e-mail: izubia@sc.ehu.es \\ ${ }^{2}$ Dept. of Systems Engineering and Automation, \\ University of the Basque Country \\ Plaza Europa 1, 20018 San Sebastián (Spain) \\ phone:+34 943 017238, fax:+34 943 017130, e-mail: xostolaza@sc.ehu.es
}

\begin{abstract}
The technical problems caused by the connection of wind farms to the electrical grid have encouraged different works in order to analyse their impact in normal situation as well as under disturbances. The most common electrical disturbances are symmetric and asymmetric short circuits that could damage both the wind farm and the network. In this case different relays in order to protect the wind farm are being developed. Islanding operation of wind farms is a particular case of abnormal situations. Nowadays, wind farms are forced to stop as soon as their disconnection is detected, but several proposals are being developed in order to maintain their operability.
\end{abstract}

This paper reports the study carried out to compare two different approaches of a frequency control for islanded wind farms. The equipment necessary to implementing each approach in a real wind farm as well as respective results are described. The strategy has been verified in a simulator implemented as a C-MEX S-Function for MATLAB, and has been applied to the wind farm of El Perdon.

\section{Key words}

Islanded operation, wind farm, transient modelling, frequency control.

\section{Introduction}

Wind farms are disconnected from the main supply due to electrical disturbances on the network. In such situation, the wind farm could be left connected to part of the utility's customer load and operate as an independent power island. But it has been observed that the main problem of islanded wind farms is the difficulty to maintain the balance between generated and demanded active and reactive power that leads to incorrect values on voltage and frequency of the islanded system [1].

Furthermore, the presence of power islands makes difficult the reconnection of the power supply network and presents a potential safety hazard. Due to these facts, nowadays, wind farms are forced to stop when the loss of mains is detected. But this practice cause the loss of the wind generation capability.

In fact, a wind farm would be able to maintain its generation capability in islanded operation if the balance of active and reactive power could be guaranteed [2]. And there will not be either problems of reconnection to the main grid because both, voltage and frequency, would have nominal values.

Several solutions have been presented to balance the active power of wind farms. The most commonly used is based on the pitch control, but its operating time is too slight for the case of islanded wind farms [2]. Another solution is called electrodynamic brake, that consist on the parallel connection or variables loads to balance the power [3].

The objective of this paper is to present an alternative strategy to the control of frequency, and analyse two possible ways of its implementation in a real wind farm sited at El Perdon.

\section{Frequency control of islanded wind farms}

Figure 1 shows the diagram of the simulator developed to analyse the impact of the wind farm of El Perdon (northern Spain) in the network. This simulator allows the calculus of symmetric and asymmetric short circuits, as well as the evolution of the wind farm in islanding operation. It has been used to compare the two possible ways of the implementation of the frequency control of islanded systems analysed in this paper.

This wind farm consist on 40 Squirrel Cage Induction Machine (SCIM) wind generators of $500 \mathrm{~kW}$, that have pitch control and an emergency braking system. A complete description and mathematical model development methodology has been reported in [4]. 


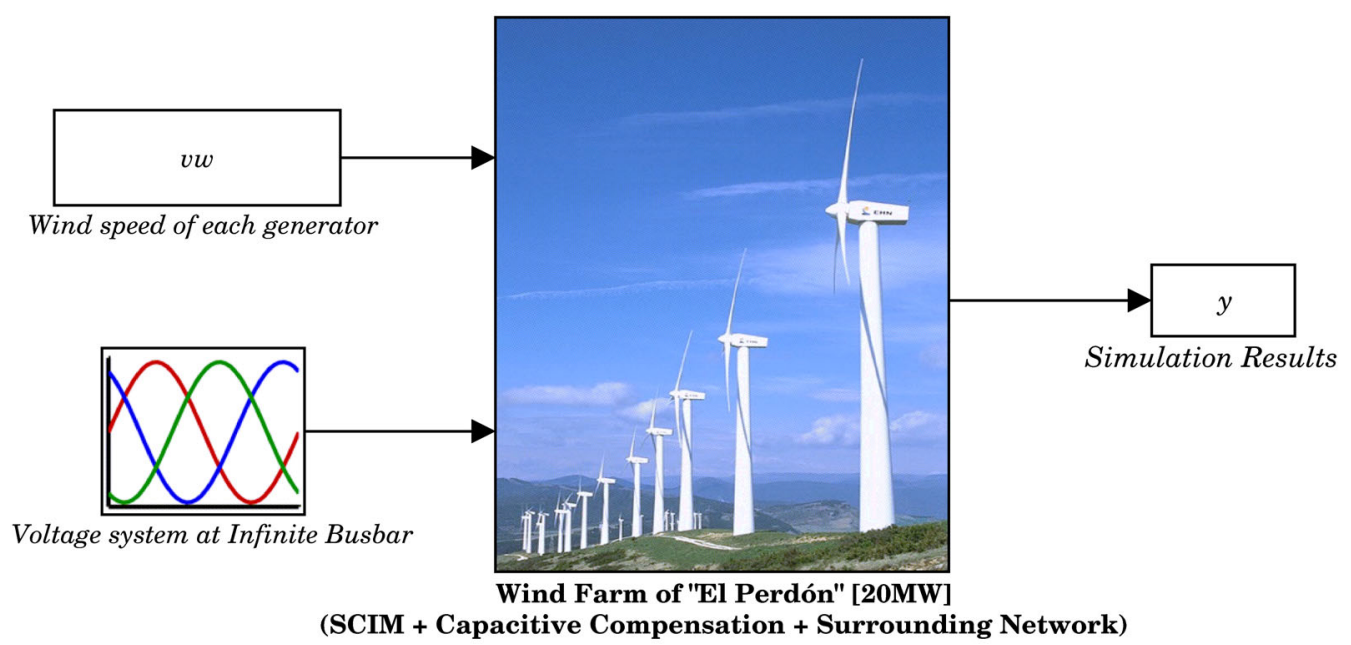

Fig. 1. Diagram of the simulator developed using MATLAB to analyse the wind farm of El Perdon.

The strategy proposed to maintain the correct value of the frequency of islanded wind farms consists on the controlled braking of wind generators. According to this strategy the modified mechanical equation of each wind generator would be,

$$
T_{e}+T_{w}-T_{B}=2 H \frac{d \omega}{d t}+D \omega
$$

where,

$T e$ is the electromagnetic torque (pu)

$T_{w}$ is the torque generated by the wind (pu)

$T_{B}$ is the braking torque (pu)

$H$ is the inertia constant of the machine (s)

$D$ is the damping coefficient (1)

$\omega$ is the angular speed of the rotor $(\mathrm{pu})$

The practical implementation of this strategy in a real wind farm shows two different possibilities: the first one consists on one central control loop that measures frequency at the common connection bar of the whole wind farm, computes the total braking torque $T_{B}^{N}$ of the wind farm in order to maintain the frequency of the overall system, and each wind generator will receive the order of applying a portion of the total braking torque,

$$
T_{B j}=\frac{T_{B}^{N}}{N}
$$

where, $N$ represents the total number of generators in the wind farm.

This implementation approach needs a communication channel between the control center and each wind generator. This communication channel already exists in most of the wind farms in order to perform Supervisory control tasks (SCADA).

Alternatively, it would be possible to measure the frequency of the system, and calculate the braking torque at each particular generator, leading to $N$ independent control loops.
Figures 2 and 3 show, respectively, block diagrams for these frequency control alternatives, where $p$ represents the Laplace variable and $s$ is the slip of generators. Note that, block diagram of Figure 3 must be repeated independently for each generator of the wind farm.

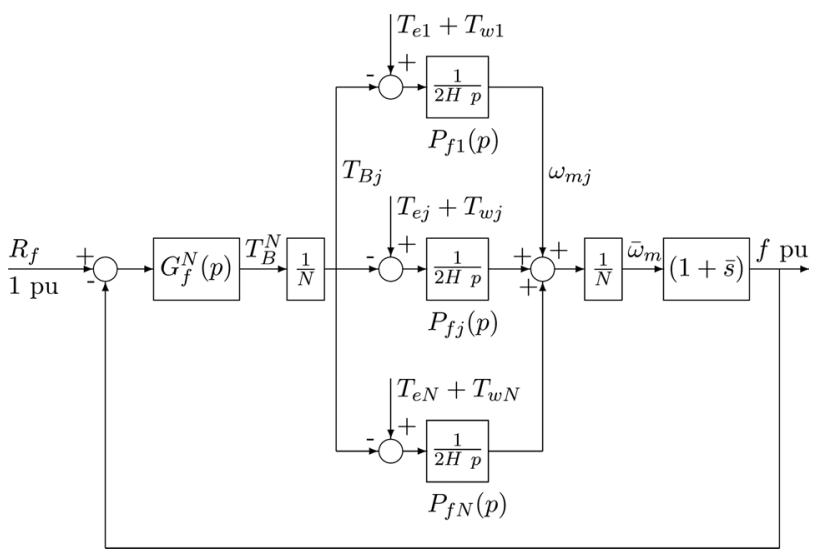

Fig. 2. Centralised frequency control structure.

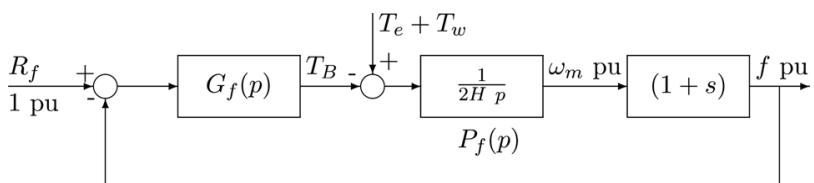

Fig. 3. Individual frequency control structure.

\section{Simulation results}

Before designing the frequency control of the wind farm the load analysis must be achieved [2]. In this analysis the installed power of the wind farm and the loads of the islanded system must be considered. If the installed power of the wind farm is able to cover the active power of loads disconnected from the main network, then with an adequate control design, the frequency of the islanded system could be maintained. 
But, on the contrary, if the wind farm can not feed all loads, some of them should be disconnected when the loss of mains happens. For this decision, technical or economical criteria could be used.

In the case of the wind farm of El Perdon studied in this work, the wind farm installed capacity can not support all three loads. In fact, only load No 1 or load No 2 [1] could be fed. In this case, based on the distance to the wind farm, the Load No1 has been selected to verify the proposed frequency control strategy.

The sequence of events simulated is:

- Disconnection of the main network

- Disconnection of Loads No 2 and No 3

- Decrease the Load No 1 until its $75 \%$

- Increase the Load No 1 until its $150 \%$

- Commute the nominal value of Load No 1

- Synchronisation with the main grid.

- Reconnection of the wind farm.

- Reconnection of Loads No 2 and No 3.

Simultaneously with this control, during the simulation, the voltage control of the islanded system has also been working.. This control calculates the reactive power necessary to asynchronous machines and local loads and maintains the voltage level of the system based on the adequate commutation of a capacitor bank.

\section{A. Simulation of $N$ control loops}

Figure 4 shows the evolution of the frequency, measured at the stator of each generator of the islanded wind farm under decentralised frequency control.

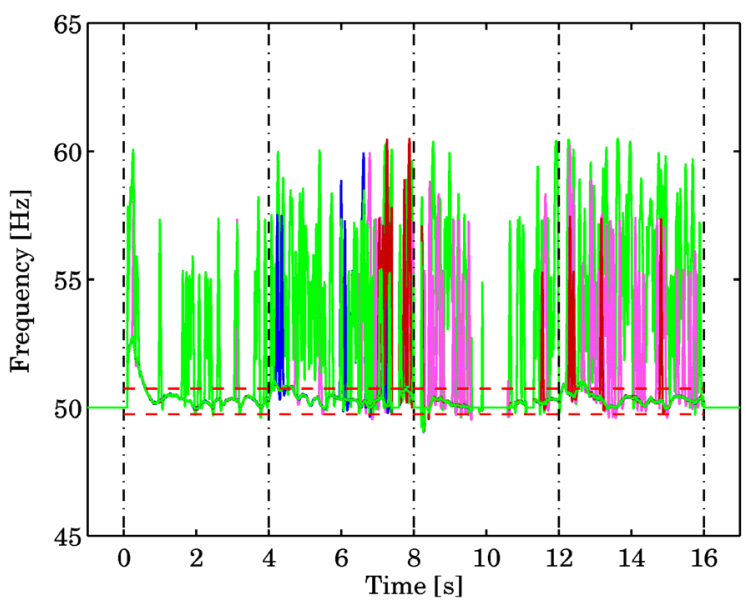

Fig. 4. Frequency of stator voltage. Decentralised control.

Figure 5 shows braking torkes computed by the controllers of each generator of the wind farm. Their noisy evolution is a consequence of the different frequency measurements at each stator. As a result, the stator mechanical speeds (Figure 6) present a too sharp evolution.

Simulation results show that the implementation based on $N$ particular control loops does not work properly. This is due to the fact that each controller tries to control the frequency of the islanded system independently, while its power generation is just a portion of the total power of the wind farm. Consequently, each generator trends to saturate, having a maximum braking torque or not torque at all. As a result, the evolution of the frequency is out of control.

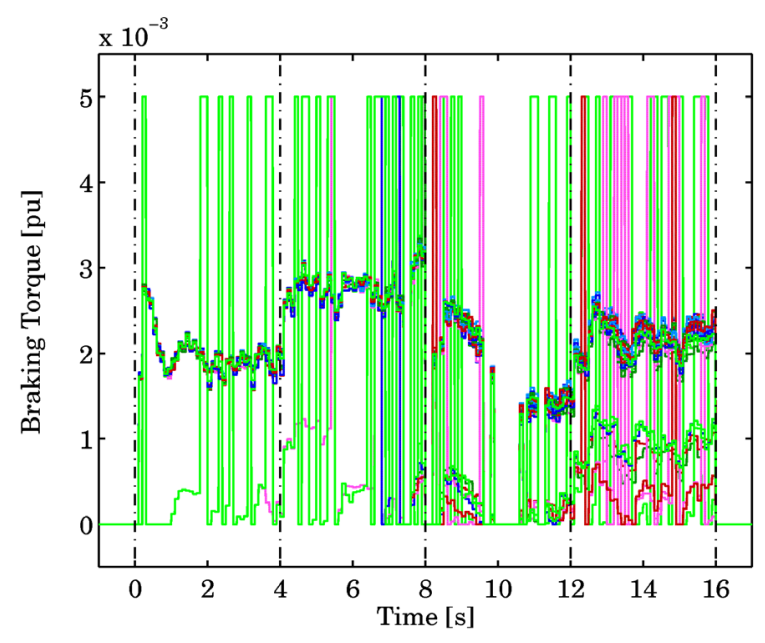

Fig. 5. Braking torque. Decentralised control scheme.

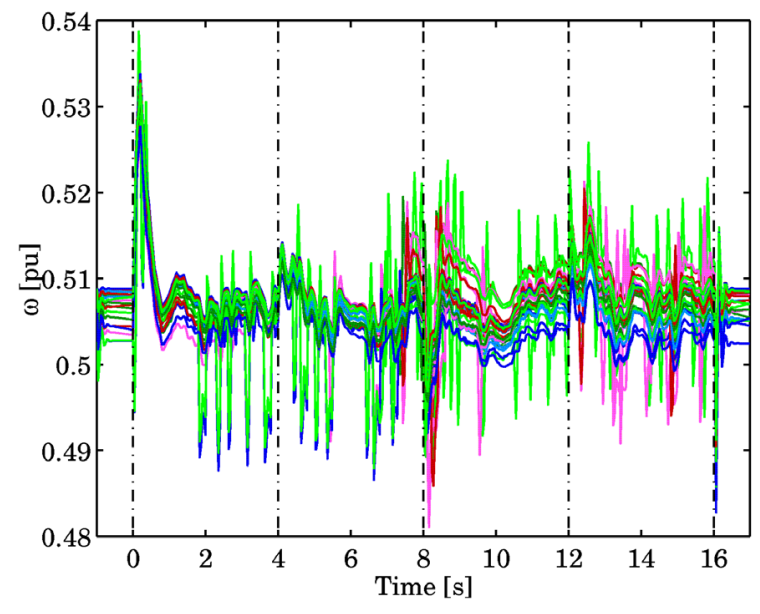

Fig. 6. Mechanical rotor speeds. Decentralised control scheme.

\section{B. Centralised control implementation}

When the frequency control is implemented by means of a centralised control structure, only a feedback loop must be closed. This controller measures frequency of voltage at the common connection point of the wind farm, and computes the total braking torque of the wind farm. The total braking torque represents a considerable fraction of the total power (typically 25\%) of the islanded network, after a convenient load analysis.

The particular braking torque for each generator is computed according to (2). This strategy trends to compensate the effect of the variability of operating conditions of generators along the wind farm. 
Figure 7 shows the evolution of the frequency of the islanded system and the total braking torque applied to the wind farm. Its behaviour is smoother than that represented in Figure 4, for the decentralised control scheme.

When the wind farm is disconnected form the network the frequency of the system goes out of limits $(50 \mathrm{~Hz}$ $\pm 1 \%$ ) but it regains its nominal value with an approximate settling time of $400 \mathrm{~ms}$.

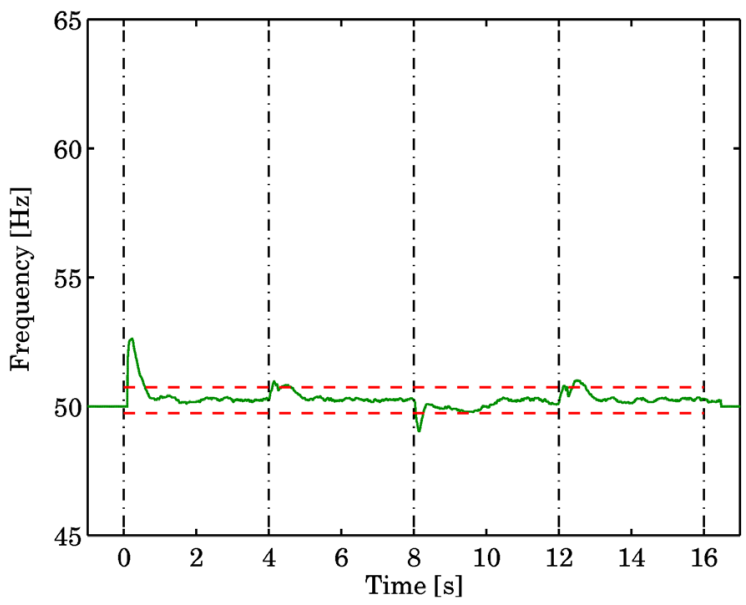

Fig. 7. Frequency of stator voltage. Centralised control scheme.

Figure 8 shows braking torque variability developed at every generator of the wind farm. When suddenly there happens an excess of generated power, the braking strategy reaches a proper frequency regulation of the islanded wind farm.

In this test, when the wind farm only feeds the Load No 1 , whose power is a half of the nominal power of the wind farm, the effect of braking torque is $0.4 \mathrm{pu}$. This effect increases to $0.5 \mathrm{pu}$ when the load decreases, and decreases to $0.2 \mathrm{pu}$ when the load is $150 \%$ of the nominal value of Load No 1.

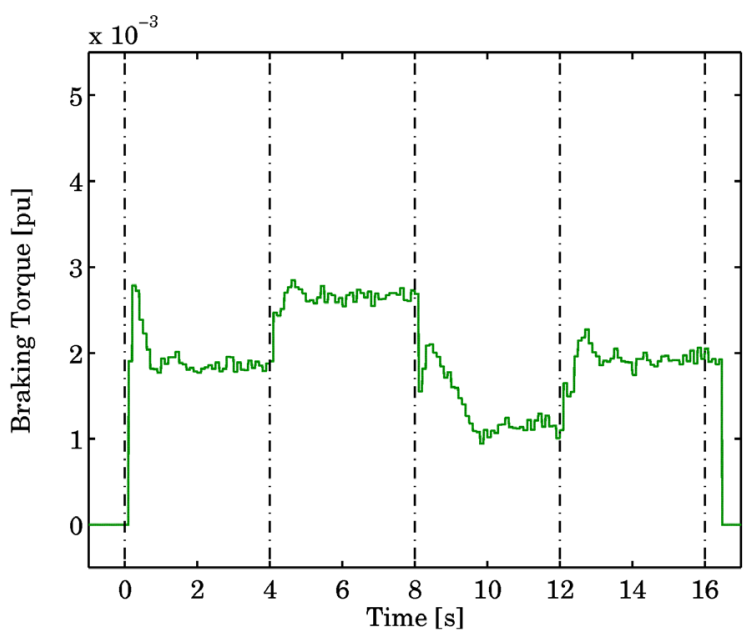

Fig. 8. Braking torque. Centralised control scheme.
The disturbance that caused the disconnection of the wind farm and surrounding loads is supposed to disappear in $t=16 \mathrm{~s}$, but the system can not be connected before it is in synchronism with the main network. When it is achieved, in $t=16.5 \mathrm{~s}$, the frequency control is disconnected and the braking signal is set to 0 .

Simulation results of this implementation approach lead to correct values of the frequency of the islanded system.

Fig. 9. shows the evolution of the mechanical rotor speed of each generator of the wind farm. Rotor speeds below 0.5 mean that generators become motors in some punctual cases. Even if all wind generators have different wind speeds, they have correct speed values and neither of them is out of control.

When the system is disconnected, loads 2 and 3 are also disconnected and the wind farm generates 9.6 MW $(0.096 \mathrm{pu})$ needed to feed load No1. In $t=4 \mathrm{~s}$ the wind farm generates only $6.75 \mathrm{MW}$, this value increases to 13 MW in $t=8 \mathrm{~s}$, and finally returns to $9.6 \mathrm{MW}$ in $t=12 \mathrm{~s}$.

In view of these results it could be concluded that the strategy adapts the power generated by the wind farm to the power demanded by variable loads of the islanded system.

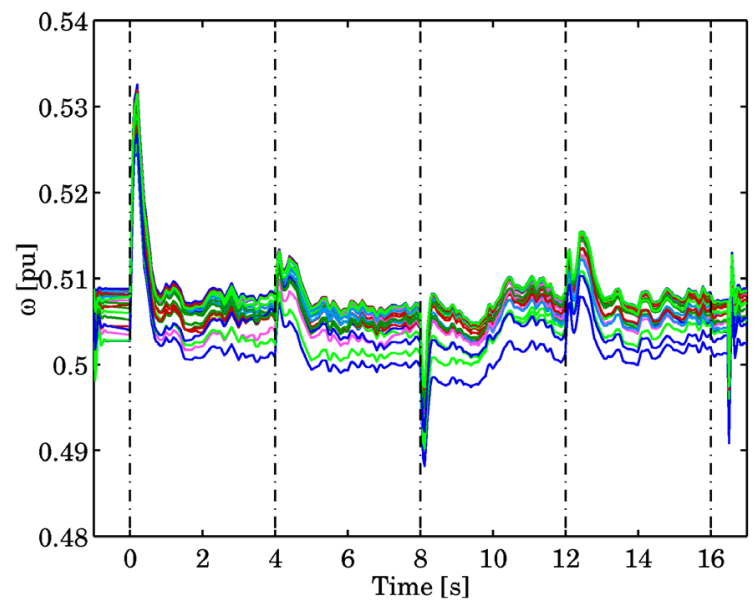

Fig. 9. Mechanical rotor speeds. Centralised control scheme.

\section{Conclusion}

An alternative frequency control strategy is presented in this paper as well as two different options of its implementation in a real wind farm. The new strategy consist on a controlled braking torque applied to each wind farm. This strategy does not ignore the pitch control, but it complements it.

Simulation results shows that the simplest at cheapest implementation that consists on one control loop connected into the control centre of the wind farm guaranties the correct evolution of the frequency of the islanded system. 


\section{Acknowledgement}

We would like to thank the electric generating company IBERDROLA S.A. and especially R. Criado, J.L. Berasategi and M. Irizar for their helpful comments and for their contribution in providing the electrical line parameters. The authors are also grateful to J. Garde, from the induction generator manufacturing company, for his contribution providing the electric machine characteristics.

\section{References}

[1] I. Zubia, X. Ostolaza, G. Tapia, A. Tapia, "Dynamic behaviour of a real wind farm in front of short-circuits and islanding operation", in Proc. ICEM2002, Vol. 1, pp. 122 and conference CD.

[2] S. Heier, Grid integration of wind energy conversion systems, John Wiley and Sons, West Sussex, England (1998).

[3] Hassan, Garrad and Ltd, " Electrodynamic braking of large wind turbines", Technical Report 1999.

[4] I. Zubia, Análisis y diseño de estrategias de operación para la explotación de parques eólicos, $\mathrm{PhD}$. Thesis (2003). 